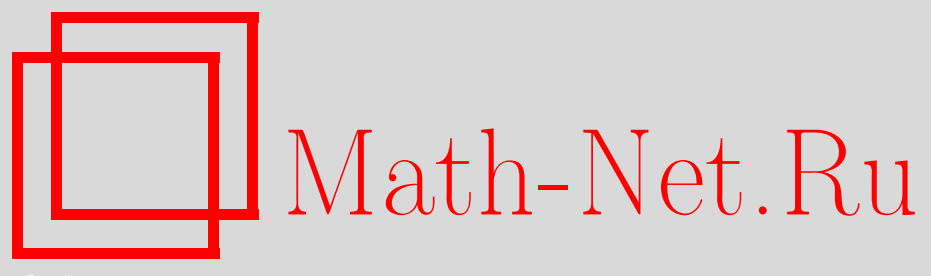

А. Г. Медведев, Гиперболические торы в системах Гамильтона с медленно меняющимся параметром, Матем. сб., 2013, том 204, номер 5, 45-66

DOI: https://doi.org/10.4213/sm8117

Использование Общероссийского математического портала Math-Net.Ru подразумевает, что вы прочитали и согласны с пользовательским соглашением http://www.mathnet.ru/rus/agreement

Параметры загрузки:

IP : 54.209 .52 .79

26 апреля 2023 г., 10:40:41

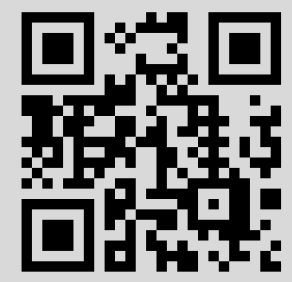




\section{А. Г. Медведев}

\section{Гиперболические торы в системах Гамильтона с медленно меняющимся параметром}

Рассматривается система Гамильтона, периодически зависящая от параметра. Предполагается, что при каждом значении параметра система имеет гиперболическое периодическое решение. Методами КАМ-теории доказывается, что если придать гамильтониану возмущение, заставив параметр меняться с малой постоянной частотой, неавтономная система будет иметь в расширенном фазовом пространстве гиперболические двумерные торы.

Библиография: 12 названий.

Ключевые слова: КАМ-теория, гиперболические торы, быстро-медленные системы.

DOI: $10.4213 / \operatorname{sm} 8117$

\section{$\S$ 1. Введение}

Рассмотрим систему Гамильтона $(M, \omega, H)$ с гамильтонианом $H(v, \tau)$, где $(M, \omega)$ - вещественно-аналитичное симплектическое многообразие размерности $2 n, v \in M$, а $\tau(\bmod 2 \pi)$ пока считается параметром. Предположим, что система обладает следующими свойствами:

- функция $H(v, \tau) 2 \pi$-периодична по параметру $\tau$ и вещественно-аналитична по всем аргументам;

- существует интервал энергии $\left(h_{1}, h_{2}\right)$ такой, что при всех $h \in\left(h_{1}, h_{2}\right)$ и всех $\tau$ система имеет периодическую гиперболическую орбиту $L_{h, \tau}$, вещественно-аналитично зависящую от $h$ и $\tau$.

Итак, имеем семейство цилиндров $N_{., \tau}$, расслоенных на периодические гиперболические орбиты:

$$
N_{\cdot, \tau}=\bigcup_{h \in\left(h_{1}, h_{2}\right)} L_{h, \tau} .
$$

Напомним определение гиперболичности.

ОПРЕДЕЛЕНИЕ 1. Периодическая орбита автономной гамильтоновой системы называется гиперболической, если все ее мультипликаторы, кроме двух тривиальных, равных единице, лежат вне единичной окружности.

Рассмотрим расширенное фазовое пространство $M \times \mathbb{T},(v, \tau) \in M \times \mathbb{T}$. Для любого значения энергии из интервала $\left(h_{1}, h_{2}\right)$ в расширенном фазовом пространстве существует двумерный тор $N_{h,}$, расслоенный на периодические решения:

$$
N_{h, \cdot}=\bigcup_{\tau \in[0,2 \pi]} L_{h, \tau} \subset M \times \mathbb{T} .
$$


ОПРЕДЕЛЕНИЕ 2. Пусть $g(h, \tau)$ - частота, соответствующая орбите $L_{h, \tau}$. Величину $\langle g(h)\rangle=\frac{1}{2 \pi} \int_{0}^{2 \pi} g(h, \tau) d \tau$ будем называть средней частотой для тоpa $N_{h, \cdot}$.

ОПРЕДЕЛЕНиЕ 3 . Пусть $\varepsilon \in\left(0, \varepsilon_{0}\right)$ - малый параметр, $g \in \mathbb{R}$. Вектор частот $(g, \varepsilon)$ называется диофантовым, если

$$
\left|g l_{1}+\varepsilon l_{2}\right| \geqslant \frac{\varepsilon}{\left(\left|l_{1}\right|+\left|l_{2}\right|\right)^{2}} \quad \forall l_{1}, l_{2} \in \mathbb{Z}, \quad\left|l_{1}\right|+\left|l_{2}\right| \neq 0 .
$$

Лемма 1 (см. [1]). Мера множества чисел $g \in[0,1]$, не удовлетворяющих системе неравенств (1.3), не превосходит 10ع.

Рассмотрим "неавтономное" возмущение исходной системы, положив $\tau=\varepsilon t$. Функция Гамильтона примет вид $H(v, \varepsilon t)$. Задачей настоящей работы является исследование существования инвариантных гиперболических двумерных торов у неавтономной системы в малой окрестности множества

$$
\tilde{N}=\bigcup_{\tau \in[0,2 \pi]} N_{\cdot, \tau} \subset M \times \mathbb{T} .
$$

Стандартными для ссылок по вопросу сохранения гиперболических диофантовых торов являются работы С. Граффа [2] и Ю. Н. Бибикова [3]. В них рассматриваются системы, имеющие гиперболические диофантовы невырожденные торы с частотами одинакового порядка, и показывается, что при малых возмущениях торы сохраняются. Особенностью нашей задачи является то, что одна частота имеет порядок возмущения, и, скорее всего, свести вопрос о существовании торов в нашей системе к стандартным теоремам невозможно.

Заметим, что если в постановке задачи убрать гиперболичность и рассмотреть двумерное многообразие $M$, то мы получим в точности задачу, решенную в статье В. И. Арнольда [1].

Системы, медленно зависящие от параметра, возникают в работах о задаче Мезера о возмущении геодезического потока на торе периодическим по времени потенциалом (см. [4]-[6]). В статье [6] методами теории инвариантных гиперболических многообразий показывается существование торов в системах вида $H(p, q)=H_{0}(p, q)+\varepsilon^{2} H_{1}(p, q, \varepsilon t)$ в предположении, что невозмущенная система имеет гиперболическое решение. В отличие от нашей задачи в [6] частота невозмущенной системы постоянна, возмущение имеет порядок $\varepsilon^{2}$ и получаемые торы, вообще говоря, не аналитичны.

Хаотическое поведение систем с медленно меняющимся параметром рассмотрено в работах [7], [8].

Сформулируем основной результат нашей статьи.

Теорема 1. Пусть для некоторого $h_{0} \in\left(h_{1}, h_{2}\right)$ выполнены следующие условия:

1) $\int_{0}^{2 \pi} \frac{\partial g}{\partial h}\left(h_{0}, \tau\right) d \tau \neq 0$

2) бункиия $H(v, \tau)$ является вещественно-аналитичной в окрестности mopa $N_{h_{0}, \cdot}$ 
Тогда существует положительная константа $\varepsilon_{0}>0$ такая, что для любого $\varepsilon \in\left(0, \varepsilon_{0}\right)$, для которого вектор частот $\left(\left\langle g\left(h_{0}\right)\right\rangle, \varepsilon\right)$ удовлетворяет системе неравенств (1.3), возмущенная неавтономная система с гамильтонианом $H(v, \varepsilon t)$ имеет двумерный инвариантный гиперболический тор с частотой $\left(\left\langle g\left(h_{0}\right)\right\rangle, \varepsilon\right)$.

Определение гиперболического тора для автономных систем содержится в работе [9]. Сформулируем аналогичное определение для неавтономного случая, когда одной из частот тора является время. Пусть $g^{t}-$ фазовый поток для некоторой неавтономной системы Гамильтона на $M \times \mathbb{T}$. Рассмотрим $l$-мерный инвариантный тор $K \subset M \times \mathbb{T}, l \leqslant n$.

ОПРЕДЕЛЕНИЕ 4. Тор $K$ называется гиперболическим, если существуют два гладких подрасслоения $E^{s, u}$ в $T_{K} M$ таких, что:

1) размерность слоев каждого подрасслоения равна $n-l+1$;

2) $E^{s, u}$ инвариантны относительно линеаризованного фазового потока, т.е.

$$
D g^{t}(w) E_{w}^{s, u}=E_{g^{t}(w)}^{s, u}, \quad w \in K, \quad t \in \mathbb{R} ;
$$

3) линеаризованный фазовый поток является сжимающим на $E^{s}$ и растягивающим на $E^{u}$, т.е. существуют положительные константы $C$ и $\lambda$, для которых выполнено

$$
\begin{gathered}
\left|D g^{t}(w)\right|_{E_{w}^{s}} \mid \leqslant C e^{-\lambda t}, \quad w \in K, \quad t \geqslant 0, \\
\left|D g^{-t}(w)\right|_{E_{w}^{u}} \mid \leqslant C e^{-\lambda t}, \quad w \in K, \quad t \geqslant 0 .
\end{gathered}
$$

Подрасслоение $E^{s}$ называется устойчивым, $E^{u}-$ неустойчивым.

Здесь и далее | | - стандартная норма матрицы, оператора или вектора в зависимости от контекста.

Оставшаяся часть статьи посвящена доказательству теоремы 1 . В $\S 2$ и $\S 3$ описано построение системы координат, в которой гамильтониан примет удобный вид для проведения КАМ-преобразований. В 33 и $§ 4$ содержится описание КАМ-процедуры.

Автор выражает благодарность своему научному руководителю Д. В. Трещеву за всестороннюю поддержку при написании настоящей работы.

\section{§ 2. Начальные преобразования}

Рассмотрим автономную систему с гамильтонианом $H(v, \tau)$. Зафиксируем некоторое $\tau_{0} \in[0,2 \pi]$. Параметру $\tau_{0}$ соответствует цилиндр из периодических гиперболических орбит $N=N_{., \tau_{0}}$ (см. (1.1)). Касательное расслоение каждой гиперболической орбиты (одномерного тора) в каждой точке $w \in N$ содержит растягивающее и сжимающее направления - $E_{w}^{u}$ и $E_{w}^{s}$ соответственно. Введем координаты в окрестности $N$, проводя рассуждения аналогично методам из работы [10].

Лемма 2. Справедливы следующие утверждения:

1) $T_{w} M=T_{w} N \oplus E_{w}^{s} \oplus E_{w}^{u}, w \in N$;

2) борма $\left.\omega\right|_{N}$ невырожденная;

3) форма $\omega$ определяет невырожденную билинейную форму на $E_{w}^{s} \oplus E_{w}^{u}$. 
ДоказАтельство. Утверждение 1) следует из того, что $\operatorname{dim} T_{w} M=2 n$, $\operatorname{dim} T_{w} N=2, E_{w}^{s} \cap E_{w}^{u}=E_{w}^{s, u} \cap T_{w} N=\{0\}$ и $\operatorname{dim} E_{w}^{u, s}=n-1$.

Утверждение 2) следует из 1 ), из невырожденности $\omega$ и из сохранения формы $\omega$ фазовым потоком. Действительно, рассмотрим произвольный вектор $\xi \neq 0, \xi \in T_{w} N$. Тогда существует такой вектор $\eta \in T_{w} M$, что $\omega(\xi, \eta) \neq 0$; $\eta=\eta_{N}+\eta_{s}+\eta_{u}$, где $\eta_{N} \in T_{w} N, \eta_{s} \in E_{w}^{s}, \eta_{u} \in E_{w}^{u}, \omega(\xi, \eta)=\omega\left(\xi, \eta_{N}\right)+\omega\left(\xi, \eta_{s}\right)+$ $\omega\left(\xi, \eta_{u}\right)$.

Из сохранения формы фазовым потоком следует $\omega\left(\xi, \eta_{s, u}\right)=\omega\left(D g^{t}(w) \xi\right.$, $\left.D g^{t}(w) \eta_{s, u}\right)=0$ (это легко получить, устремляя $t$ к $\pm \infty$ : учитывая неравенства в определении гиперболического тора, получим в пределе $\omega(\cdot, 0)=0)$. Следовательно, $\omega(\xi, \eta)=\omega\left(\xi, \eta_{N}\right) \neq 0$, т.е. форма $\left.\omega\right|_{N}$ невырожденная.

Утверждение 3) доказывается аналогично 2). Рассмотрим произвольный вектор $v_{u} \neq 0, v_{u} \in E_{w}^{u}$. Существует такой вектор $\beta \in T_{w} M$, что $\omega\left(v_{u}, \beta\right) \neq 0$, $\beta=\beta_{N}+\beta_{s}+\beta_{u}$, где $\beta_{N} \in T_{w} N, \beta_{s} \in E_{w}^{s}, \beta_{u} \in E_{w}^{u}$. Тогда $\omega\left(v_{u}, \beta\right)=\omega\left(v_{u}, \beta_{N}\right)+$ $\omega\left(v_{u}, \beta_{s}\right)+\omega\left(v_{u}, \beta_{u}\right)$. Из сохранения формы фазовым потоком следует, что $\omega\left(v_{u}, \beta_{N}\right)=\omega\left(v_{u}, \beta_{u}\right)=0$, откуда получаем $\omega\left(v_{u}, \beta_{s}\right) \neq 0$. Лемма доказана.

Из утверждения 2) леммы 2 вытекает, что $\left(N,\left.\omega\right|_{N}\right)$ - симплектическое многообразие размерности 2. Введем на $N$ переменные действие-угол $(y, x)$ :

$$
\left.H\right|_{N}=\left.H\right|_{N}(y),\left.\quad \omega\right|_{N}=d y \wedge d x, \quad x=x(\bmod 2 \pi) .
$$

Расслоение $T_{N} M$ тривиальное, т.е. $T_{N} M=N \times \mathbb{R}^{2 n}$. Из утверждения 3) леммы 2 следует, что на $E_{w}^{s} \oplus E_{w}^{u}$ можно ввести координаты $\left(z_{s}, z_{u}\right)$, в которых $\left.\omega\right|_{E_{w}^{s} \oplus E_{w}^{u}}=d z_{s} \wedge d z_{u}$.

Действительно, выберем на $E_{w}^{s}=\mathbb{R}^{n-1}$ произвольные аффинные координаты. Они порождают координаты на $E^{s^{*}}, e_{i} \mapsto \omega\left(\cdot, e_{i}\right)$, а последние порождают координаты на $E_{w}^{u}, \eta_{j}: \omega\left(\eta_{j}, e_{i}\right)=\delta_{i j}$.

Вспомним, что на $N$ система координат уже задана. Перенесем координаты в окрестность подмногообразия $N$ при помощи экспоненциального отображения.

Фиксируем риманову метрику на $M$. Пусть $\eta$ - точка в малой окрестности $N$. Проведем из $\eta$ на $N$ кривую минимальной длины $l_{\eta}$, параметризуем ее параметром, пропорциональным натуральному, так, что координата точки $l_{\eta} \cap N$ равна 0 , а координата $\eta$ равна 1 . В качестве первой пары координат возьмем координаты $(y, x)$ точки пересечения кривой с $N$, в качестве второй пары $\left(z_{s}, z_{u}\right)$ возьмем координаты проекции вектора скорости $l_{\eta}$ в точке $l_{\eta} \cap N$ на $E_{w}^{s} \oplus E_{w}^{u}$.

Итак, в малой окрестности $N$ заданы координаты $\left(y, x, z_{s}, z_{u}\right)$, в которых симплектическая структура имеет вид

$$
\omega=d y \wedge d x+d z_{s} \wedge d z_{u}+O\left(z_{s}, z_{u}\right) .
$$

Продеформируем их до канонических при помощи теоремы Дарбу (см. [11], а также [12]).

Теорема 2 (теорема Дарбу). Пусть $M$ - четномерное многообразие, $N$ подмногообразие, $\omega, \Omega$ - две симплектические структуры на $M$, ограничения которых на $N$ совпадают. Предположим, что $\omega$ и $\Omega$ непрерывно деформируются друг в друга в классе симплектических структур на $M$, совпадающих на $N$. Тогда существуют окрестности $U_{0}$ и $U_{1}$ подмногообразия $N$ в $M u$ 
диффеоморфизм $g: U_{0} \mapsto U_{1}$, тождественный на $N$, который переводит $\left.\Omega\right|_{U_{1}}$ в $\left.\omega\right|_{U_{0}}: g^{*} \Omega=\omega$.

В нашей задаче $N$ - подмногообразие $M$, формы $\omega=d y \wedge d x+d z_{s} \wedge d z_{u}+$ $O\left(z_{s}, z_{u}\right)$ и $\Omega=\left.\omega\right|_{N}=d y \wedge d x+d z_{s} \wedge d z_{u}$ гомотопны, т.е. существует гладкое семейство невырожденных замкнутых форм

$$
\omega(t)=(1-t) \omega+t \Omega, \quad \omega(0)=\omega, \quad \omega(1)=\Omega .
$$

Согласно теореме Дарбу в окрестности $N$ существует система координат $\left(\widehat{y}, \widehat{x}, \widehat{z_{s}}, \widehat{z_{u}}\right)$, в которой $\omega=d \widehat{y} \wedge d \widehat{x}+d \widehat{z_{s}} \wedge d \widehat{z_{u}}$. Выберем эти канонические координаты. Для простоты уберем крышки над обозначениями. В нашем случае диффеоморфизм из теоремы Дарбу будет вещественно-аналитичным. Для доказательства этого факта достаточно предположить в условиях теоремы вещественно-аналитичность многообразий и симплектических структур и повторить доказательство теоремы Дарбу из [12].

В окрестности цилиндра $N=\left\{z_{u}=z_{s}=0\right\}$ запишем разложение гамильтониана в ряд Тейлора:

$$
\begin{aligned}
H=F\left(y, \tau_{0}\right) & +\left\langle A_{u}\left(y, x, \tau_{0}\right), z_{u}\right\rangle+\left\langle A_{s}\left(y, x, \tau_{0}\right), z_{s}\right\rangle+\left\langle z_{s}, C\left(y, x, \tau_{0}\right) z_{u}\right\rangle \\
& +\left\langle z_{u}, B_{u}\left(y, x, \tau_{0}\right) z_{u}\right\rangle+\left\langle z_{s}, B_{s}\left(y, x, \tau_{0}\right) z_{s}\right\rangle+O_{3}\left(z_{s}, z_{u}\right),
\end{aligned}
$$

где $A_{u}, A_{s}$ - векторы размерности $n-1$, а $C, B_{s}, B_{u}$ - матрицы порядка $n-1$, функция $F\left(y, \tau_{0}\right)=H\left(y, x, 0,0, \tau_{0}\right)$ не зависит от $x$ (см. (2.1)). Не ограничивая общности, будем считать, что $\partial F(y, \tau) / \partial y>0$ для всех $\tau$. Поскольку по определению гиперболичности многообразия $\left\{y=\right.$ const, $\left.z_{s}=0\right\}$ и $\{y=$ const, $\left.z_{u}=0\right\}$ инвариантны относительно линеаризованного фазового потока, то канонические уравнения на $z_{u}$ и $z_{s}$ должны иметь вид

$$
\dot{z}_{u}=A_{1}\left(y, x, \tau_{0}\right) z_{u}+O_{2}\left(z_{s}, z_{u}\right), \quad \dot{z}_{s}=A_{2}\left(y, x, \tau_{0}\right) z_{s}+O_{2}\left(z_{s}, z_{u}\right),
$$

где $A_{1}\left(y, x, \tau_{0}\right)$ и $A_{2}\left(y, x, \tau_{0}\right)$ - матрицы порядка $n-1$. Следовательно, $A_{s}=$ $A_{u}=0, B_{s}=B_{u}=0$ и функция Гамильтона принимает вид

$$
H\left(y, x, z_{s}, z_{u}, \tau_{0}\right)=F\left(y, \tau_{0}\right)+\left\langle z_{s}, C\left(y, x, \tau_{0}\right) z_{u}\right\rangle+O_{3}\left(z_{s}, z_{u}\right) .
$$

При рассмотрении свойств матрицы $C$ нам понадобится понятие $\nu$-положительной определенности для матриц, введенное в работе [9]. Пусть задана вещественная квадратная матрица $Q=Q(s)$ порядка $l, s \in \mathbb{T}^{k}, \nu \in \mathbb{R}^{k}$ - постоянный вектор частот. Рассмотрим систему уравнений

$$
\dot{s}=\nu, \quad \dot{u}=Q(s) u, \quad s \in \mathbb{T}^{k}, \quad u \in \mathbb{R}^{l} .
$$

Пусть $\phi_{t}-$ фундаментальная матрица этой системы, т.е. $\phi_{0}=I$ и решение системы с начальными условиями $s(0)=s_{0}, u(0)=u_{0}$ имеет вид $s(t)=s_{0}+\nu t$, $u(t)=\phi_{t}\left(s_{0}\right) u_{0}$.

ОПРЕДЕЛЕНИЕ 5. Матрица $Q(s)$ называется $\nu$-положителъно определенной, если существуют положительные константы $\Lambda, \lambda$ такие, что

$$
\left|\phi_{-t}\right| \leqslant \Lambda e^{-\lambda t}, \quad s \in \mathbb{T}^{k}, \quad t \geqslant 0 .
$$

Матрица $Q(s)$ называется $\nu$-отрицательно определенной, если $\left|\phi_{t}\right| \leqslant \Lambda e^{-\lambda t}$, $t \geqslant 0$. 
Лемма 3. Для каждого фиксированного $y=y_{0}$ матрица $C\left(y_{0}, x, \tau_{0}\right)$ является $\nu$-положительно определенной с вектором частот $\nu=(\omega, 0)$, где $\omega$ произвольное положительное число.

ДоказАТЕЛЬСтво. Обозначим $\omega_{0}=\partial F\left(y_{0}, \tau_{0}\right) / \partial y$ и запишем уравнения в вариациях:

$$
\dot{x}=\omega_{0}, \quad \dot{y}=0, \quad \dot{\xi}_{u}=C\left(y_{0}, x, \tau_{0}\right) \xi_{u}, \quad \dot{\xi}_{s}=-C^{T}\left(y_{0}, x, \tau_{0}\right) \xi_{s}, \quad \dot{\tau}_{0}=0 .
$$

Поскольку $z_{u}$ в линейном приближении - координата на $E^{u}$, то в силу условий гиперболичности будет выполнено $\left|\xi_{u}(-t)\right| \leqslant \Lambda e^{-\lambda t}, t \geqslant 0,\left|\xi_{u}(0)\right|=1$ для некоторых положительных констант $\Lambda$ и $\lambda$. Остается заметить, что свойство $\nu$-положительной определенности не меняется при умножении матрицы на положительную константу. Лемма доказана.

Лемма 4. В некоторой окрестности $\widetilde{N}$ (см. (1.4)) существует периодичное по параметру $\tau$ вещественно-аналитичное каноническое преобразование $g_{\tau}$, приводящее систему с гамильтонианом $H(v, \tau) \kappa$ виду (2.2).

ЗАмЕЧАниЕ 1. Здесь и далее вещественно-аналитичность предполагается в том числе по $\tau$.

ДокАЗАТЕЛЬСТво ЛЕммы 4. Повторим ход рассуждений из $\S 1$. Зафиксируем на $M$ метрику. По условию мы имеем параметризованное по параметру $\tau(\bmod 2 \pi)$ семейство цилиндров $N_{\tau}$. Выберем на цилиндрах семейство образующих. Используя эти образующие в качестве отсчета угловых переменных и учитывая то, что переменные действие-угол в двумерном случае вводятся явными формулами, заключаем, что координаты $(y, x)$ на $N_{\tau}$ можно ввести вещественно-аналитично и периодично по параметру $\tau$.

Координаты на $E_{w}^{s}$ и $E_{w}^{u}(w \in N)$ могут быть выбраны вещественно-аналитичными и периодичными по $\tau$. Это следует из вещественно-аналитичности гамильтониана и семейства цилиндров $N_{\tau}$.

Как и в $\S 1$, перенесем координаты в окрестности $N_{\tau}$ при помощи экспоненциального отображения.

Итак, мы получили семейство замен, приводящих форму к виду

$$
\omega=d y \wedge d x+d z_{s} \wedge d z_{u}+O\left(z_{s}, z_{u}\right)
$$

Воспользуемся теоремой Дарбу для получения окончательных преобразований. Важно, чтобы диффеоморфизм из теоремы Дарбу был вещественно-аналитичным и периодичным по параметру. Добавим в условия теоремы вещественноаналитичную и периодичную зависимость от параметра и в остальном повторим доказательство теоремы Дарбу из [12]. Лемма доказана.

Так как $\widetilde{N}$ - произведение цилиндра и окружности, то симплектическая структура точна в окрестности этого множества, т.е. существует 1-форма $\omega^{1}$ : $\omega=d \omega^{1}$.

Из определения канонической замены координат следует существование функции $S\left(y, x, z_{s}, z_{u}, \tau\right)$ и нового гамильтониана $H\left(y, x, z_{s}, z_{u}, \tau\right)$ таких, что

$$
\omega^{1}-H(v, \tau) d t=y(v, \tau) d x+z_{s}(v, \tau) d z_{u}-H\left(y, x, z_{s}, z_{u}, \tau\right) d t+d S .
$$


Положим $\tau=\varepsilon t$. Тогда

$$
d S=\left.d S\right|_{\tau=\text { const }}+\varepsilon \frac{\partial S}{\partial \tau} d t
$$

Частные производные по $y, x, z_{s}, z_{u}$ у функции $S$ определены однозначно, вещественно-аналитично и периодично по $\tau$. Следовательно, $\partial S / \partial \tau$ с точностью до слагаемого, зависящего только от $\tau$, обладает этими свойствами.

Полученная замена приводит неавтономный гамильтониан $H(v, \varepsilon t)$ к виду

$$
H\left(y, x, z_{s}, z_{u}, \varepsilon t\right)=F(y, \varepsilon t)+\left\langle z_{s}, C(y, x, \varepsilon t) z_{u}\right\rangle+O(\varepsilon)+O_{3}\left(z_{s}, z_{u}\right),
$$

где все функции вещественно-аналитичны по своим аргументам и $2 \pi$-периодичны по $\tau=\varepsilon t$ и $x$.

ЗАмечАниЕ 2. Не ограничивая общности, можно считать, что $h_{0}=0$. Мы всегда можем прибавить к гамильтониану такую функцию времени, что нулевой уровень энергии будет соответствовать нулевому значению координаты $y$, $h_{0}=F(0, \varepsilon t)=0$.

Будем рассматривать поведение системы в окрестности $y=0, z_{u}=z_{s}=0$. Из выполнения условий 1) и 2) теоремы 1 следуют неравенство для частоты $g(0, \tau)=\partial F(0, \tau) / \partial y \neq 0$ и условие невырожденности

$$
\int_{0}^{2 \pi} \frac{\partial F^{2}}{\partial y^{2}}(0, \tau) d \tau \neq 0
$$

Не ограничивая общности, положим $g(0, \tau)>0$ для всех $\tau$.

\section{§ 3. Дополнительные преобразования координат}

Цель этого параграфа - провести серию замен переменных, после которых гамильтониан примет вид, удобный для проведения KAM-процедуры, т.е. получить канонические координаты, в которых частоты не зависят от угловых координат и времени в нулевом приближении по $y, z_{s}, z_{u}$. Воспользуемся техникой из статьи [1].

Запишем интегральный инвариант:

$$
y d x+z_{s} d z_{u}-H d t=-\frac{1}{\varepsilon} H d \tau+z_{s} d z_{u}-(-y) d x,
$$

введем новый гамильтониан $Y$ и координаты

$$
P=-\frac{1}{\varepsilon} H, \quad Q=\tau, \quad Z_{u}=z_{u}, \quad Z_{s}=z_{s}, \quad T=x, \quad Y=-y .
$$

Разрешая уравнение (2.3) относительно $y$, найдем $y=y\left(x, H, z_{s}, z_{u}, \tau, \varepsilon\right)$. Имеем

$$
F(y, \tau)=H-\left\langle z_{s}, C(0, x, \tau) z_{u}\right\rangle+O(\varepsilon)+O_{3}\left(y, z_{s}, z_{u}\right) .
$$

Замечание 2 и теорема о неявной функции позволяют утверждать, что существует обратная функция $F^{-1}(\cdot, \tau)$,

$$
y=F^{-1}(H, \tau)-\frac{\partial F^{-1}}{\partial H}(0, \tau)\left\langle z_{s}, C(0, x, \tau) z_{u}\right\rangle+O(\varepsilon)+O_{3}\left(y, z_{s}, z_{u}\right) .
$$


Обозначим

$$
\begin{gathered}
G(\varepsilon P, Q)=-F^{-1}(H, \tau), \quad \widetilde{C}(Q, T)=\frac{\partial F^{-1}}{\partial H}(0, \tau) C(0, x, \tau), \\
O(\varepsilon)=\varepsilon H_{1}\left(\varepsilon P, Q, Z_{s}, Z_{u}, T\right)
\end{gathered}
$$

где функция $H_{1} 2 \pi$-периодическая по $Q$ и $T$. Гамильтониан в новых переменных имеет вид

$$
Y=G(\varepsilon P, Q)+\left\langle Z_{s}, \widetilde{C}(Q, T) Z_{u}\right\rangle+\varepsilon H_{1}\left(\varepsilon P, Q, Z_{s}, Z_{u}, T\right)+O_{3}\left(\varepsilon P, Z_{s}, Z_{u}\right) .
$$

Остановимся подробнее на свойствах функции $G$ и матрицы $\widetilde{C}$. Из замечания 2 следует, что

$$
\frac{\partial G}{\partial \varepsilon P}(0, Q)=\frac{1}{g(0, Q)} .
$$

Справедливо соотношение

$$
\widetilde{C}(Q, T)=\frac{\partial F^{-1}}{\partial H}(0, \tau) C(0, x, \tau) .
$$

Заметим, что координаты $z_{u}$ и $z_{s}$ являются в линейном приближении координатами на $E^{u}$ и $E^{s}$ (теорема Дарбу сохраняет это свойство, а последняя замена тождественна для этих координат), поэтому матрица $\widetilde{C}(Q, T) \nu$-положительно определена с вектором частот $\nu=(0,1)$ (см. лемму 3$)$. Для упрощения записей уберем волну над $C$.

Перейдем к переменным $\left(P^{\prime}, Q, Z_{s}, Z_{u}, T\right)=\left(\varepsilon P, Q, Z_{s}, Z_{u}, T\right)$, при этом симплектическая структура в новых координатах имеет вид

$$
\omega=\frac{1}{\varepsilon} d P^{\prime} \wedge d Q+d Z_{s} \wedge d Z_{u} .
$$

Сделаем еще одну замену: $\left(P^{\prime}, Q, Z_{s}, Z_{u}, T\right) \mapsto\left(I, X, Z_{s}, Z_{u}, T\right)$, где $(I, X)-$ переменные действие-угол для $G\left(P^{\prime}, Q\right)$. Пусть $S=s(I, Q)+Z_{s} Z_{u}$ - производящая функция искомой замены. Тогда

$$
\begin{gathered}
G\left(\frac{\partial s}{\partial Q}(I, Q), Q\right)=K(I), \quad \frac{\partial s}{\partial Q}(I, Q)=G^{-1}(K(I), Q)=-F(-K(I), Q), \\
s(I, Q)=-\int_{0}^{Q} F(-K(I), Q) d Q .
\end{gathered}
$$

Лемма 5. Существует гладкая функиия $K(I)$, обладающая (в некоторой малой окрестности $I=0)$ следующими свойствами:

1) $-\int_{0}^{2 \pi} F(-K(I), Q) d Q=2 \pi I$

2) $d K(0) / d I=1 /\langle g(0)\rangle$;

3) $d^{2} K(0) / d I^{2} \neq 0$.

ДокАЗАтельство. Рассмотрим функцию

$$
\langle\widetilde{F}\rangle(y)=-\frac{1}{2 \pi} \int_{0}^{2 \pi} F(-y, Q) d Q .
$$


Из свойств функции $F$ следует, что

$$
\frac{d\langle\widetilde{F}\rangle}{d y}(0)=\langle g(0)\rangle \neq 0, \quad \frac{d^{2}\langle\widetilde{F}\rangle}{d y^{2}}(0) \neq 0,
$$

где $\langle g(0)\rangle \neq 0$ - средняя частота для тора $N_{0}$ (см. определение 2). Применяя теорему об обратной функции, получим утверждения леммы, взяв $K(\cdot)=$ $\langle\widetilde{F}\rangle^{-1}(\cdot)$. Лемма доказана.

На этом введение системы координат, удобных для проведения KAM-преобразований, закончено. Перепишем гамильтониан и симплектическую структуру:

$$
\begin{gathered}
H=K(I)+\left\langle Z_{s}, C(X, T) Z_{u}\right\rangle+\varepsilon H_{1}\left(I, X, Z_{s}, Z_{u}, T\right)+O_{3}\left(I, Z_{s}, Z_{u}\right), \\
\omega=\frac{1}{\varepsilon} d I \wedge d X+d Z_{s} \wedge d Z_{u} .
\end{gathered}
$$

При $\varepsilon H_{1}=0$ система имеет инвариантный тор $\left\{Z_{u}=Z_{s}=0, I=0\right\}$. Далее проводится серия KAM-замен. В ходе каждой замены квадратично повышается порядок малости соответствующих членов разложения функции $H_{1}$ в ряд Тейлора.

После проведения КАМ-процедуры мы получим предельную систему координат $\left(I_{\infty}, X_{\infty}, Z_{s \infty}, Z_{u \infty}, T\right)$. Будет показано, что $I=I_{\infty}+O(\varepsilon)$. Последнее соотношение обеспечивает сохранение малости преобразований при переходе к стандартной симплектической структуре.

\section{§ 4. Вспомогательные утверждения к проведению КАМ-преобразований}

Представим выражение (3.2) в следующем виде:

$$
H=k_{1} I+k_{2} I^{2}+\left\langle Z_{s}, C Z_{u}\right\rangle+\Phi+\Omega+\Psi+F,
$$

где

$$
\begin{gathered}
F=O_{3}\left(I, Z_{s}, Z_{u}\right), \quad C=C(X, T) \\
\Phi=h(X, T, \varepsilon)+p(X, T, \varepsilon) I+q(X, T, \varepsilon) I^{2}, \\
\Omega=A(X, T, \varepsilon) Z_{u}+B(X, T, \varepsilon) Z_{s}+\left\langle Z_{u}, L(X, T, \varepsilon) Z_{u}\right\rangle \\
+\left\langle Z_{s}, K(X, T, \varepsilon) Z_{s}\right\rangle+\left\langle Z_{s}, R(X, T, \varepsilon) Z_{u}\right\rangle \\
\Psi=D(X, T, \varepsilon) I Z_{u}+Q(X, T, \varepsilon) I Z_{s},
\end{gathered}
$$

функции $h, p, q, A, B, D, R, L, K$ вещественно-аналитичны и имеют порядок малости $O(\varepsilon), k_{1}$ и $k_{2}$ - константы.

ЗАмЕчАниЕ 3. Для краткости записей далее мы не будем указывать аргументы у функций.

Для дальнейших рассуждений понадобятся следующие обозначения и утверждения.

Символом $D_{\rho}$ будем обозначать комплексную полосу ширины $\rho$ :

$$
D_{\rho}=\left\{\eta \in \mathbb{C}^{2}:\left|\operatorname{Im} \eta_{j}\right| \leqslant \rho, j=1,2\right\} .
$$


Символом $B_{s}^{k}$ обозначим шар радиуса $s$ в $\mathbb{C}^{k}$ :

$$
B_{s}^{k}=\left\{z \in \mathbb{C}^{k}:\left|z_{j}\right| \leqslant s, j=1, \ldots, k\right\} .
$$

Символом $|\cdot|_{\rho}$ обозначим $C^{0}$-норму в соответствующей области или полосе, символом $\langle\cdot\rangle$ - среднее значение периодической функции.

ЗАмЕчаниЕ 4. Из леммы 5 следуют условия невырожденности $k_{1} \neq 0$, $k_{2} \neq 0$. Без ограничения общности можно считать, что $k_{1}=1 /\langle g(0)\rangle=1$.

ЗАмЕчАниЕ 5. Формулировка теоремы 1 предполагает диофантовость вектора $(\varepsilon, 1)$, что эквивалентно выполнению неравенств

$$
\left|\varepsilon l_{1}+l_{2}\right| \geqslant \frac{\varepsilon}{\left(\left|l_{1}\right|+\left|l_{2}\right|\right)^{2}} \quad \forall l_{1}, l_{2} \in \mathbb{Z}, \quad\left|l_{1}\right|+\left|l_{2}\right| \neq 0 .
$$

Так как исходный гамильтониан вещественно-аналитичен, существует комплексная полоса $D_{\rho}, \rho<1$, в которой функции $h, p, q, A, B, D, R, L, K$ аналитичны и ограничены по модулю некоторой константой $M$. За счет выбора $\varepsilon$ константу $M$ можно сделать сколь угодно малой.

Рассмотрим малую комплексную окрестность $B_{\rho}^{2 n-1}=\left\{|I|<\rho,\left|Z_{u}\right|<\rho\right.$, $\left.\left|Z_{s}\right|<\rho\right\}$. Функция $F\left(I, Z_{s}, Z_{u}, X, T\right)$ ограничена в области $\Delta_{\rho}=D_{\rho} \times B_{\rho}^{2 n-1}$. Из свойства $F=O_{3}\left(I, Z_{s}, Z_{u}\right)$ следует существование положительной константы $M^{f}$ такой, что

$$
|F| \leqslant M^{f} \sum_{\substack{i+j+k=3 \\ i, j, k \geqslant 0}}|I|^{i}\left|Z_{s}\right|^{j}\left|Z_{u}\right|^{k}, \quad\left(I, X, Z_{s}, Z_{u}, T\right) \in \Delta_{\rho} .
$$

Напомним две стандартные леммы.

Лемма 6. Пусть функиия $\varphi: D_{\rho} \rightarrow \mathbb{C}$ аналитична, $2 \pi$-периодична и $\|\varphi\|_{\rho} \leqslant M$. Тогда коэфбициенты ряда Фуръе $\varphi(\eta)=\sum_{m \in \mathbb{Z}^{2}} \varphi_{m} e^{i\langle m, \eta\rangle}$ удовлетворяют оченкам

$$
\left|\varphi_{m}\right| \leqslant M e^{-\left(\left|m_{1}\right|+\left|m_{2}\right|\right) \rho}, \quad m=\left(m_{1}, m_{2}\right) .
$$

ЛЕмма 7 (оценки Коши). Пусть функиия $g: B_{s}^{1} \rightarrow \mathbb{C}$ аналитична в шаре $B_{s}^{1} \subset \mathbb{C}$, и пусть $|g| \leqslant G$ в $B_{s}^{1}$. Тогда для любого положительного $u<s u$ любого $l \in \mathbb{N}$ производнъе $g^{(l)}=d^{l} g / d z^{l}$ в шаре $B_{s-u}^{1}$ удовлетворяют оценкам

$$
\left|g^{(l)}(z)\right| \leqslant \frac{l ! G}{u^{l}}
$$

Лемма 8. Рассмотрим периодичную вещественно-аналитичную функцию $h(X, T)$ с нулевым средним и диофантов вектор частот $(\varepsilon, 1)$. Предположим, что $h$ аналитична в полосе $D_{\rho} u|h| \leqslant M$.

Тогда существует функиия $\psi(X, T)$, обладающая в $D_{\rho_{1}}, \rho_{1}=\rho-2 \sigma>0$, следующими свойствами:

1) $\psi$ удовлетворяет уравнению

$$
\varepsilon \frac{\partial \psi}{\partial X}+\frac{\partial \psi}{\partial T}=-h(X, T)
$$

2) в полосе $D_{\rho_{1}}$ функиия $\psi$ аналитична; 
3) справедливы оценки

$$
|\psi|_{\rho_{1}} \leqslant \frac{17 M}{\varepsilon \sigma^{4}}, \quad\left|\frac{\partial \psi}{\partial X}\right|_{\rho_{1}} \leqslant \frac{17 M}{\varepsilon \sigma^{5}}, \quad\left|\frac{\partial \psi}{\partial T}\right|_{\rho_{1}} \leqslant \frac{17 M}{\varepsilon \sigma^{5}} .
$$

ДокАЗАТЕЛЬСтво. Представим функции в виде рядов Фурье:

$$
h(X, T)=\sum_{(k, l) \neq(0,0)} h_{k l} e^{i(k X+l T)}, \quad \psi(X, T)=\sum_{(k, l) \neq(0,0)} \psi_{k l} e^{i(k X+l T)} .
$$

Подставляя ряды в уравнение (4.2), получаем

$$
\psi_{k l}=-\frac{h_{k l}}{i(\varepsilon k+l)}, \quad k^{2}+l^{2} \neq 0 .
$$

Полагая $\psi_{00}=0$, имеем

$$
\psi(X, T)=-\sum_{(k, l) \neq(0,0)} \frac{h_{k l}}{i(\varepsilon k+l)} e^{i(k X+l T)} .
$$

Из ограниченности $h$ в полосе $D_{\rho}$ и леммы 6 следует неравенство

$$
\left|h_{k l}\right| \leqslant M e^{-(|k|+|l|) \rho} .
$$

Учитывая условия диофантовости, для любого $z=\left(z_{1}, z_{2}\right) \in D_{\rho-\sigma}$ имеем

$$
|\psi(z)| \leqslant \sum_{(k, l) \neq(0,0)}\left|\psi_{k l} e^{i\left(k z_{1}+l z_{2}\right)}\right| \leqslant \sum_{(k, l) \neq(0,0)} \frac{M}{\varepsilon}(|k|+|l|)^{2} e^{-(|k|+|l|) \sigma} .
$$

Справедлива интегральная оценка суммы ряда

$$
|\psi|_{\rho-\sigma} \leqslant \sum_{(k, l) \neq(0,0)} \frac{M}{\varepsilon}(|k|+|l|)^{2} e^{-(|k|+|l|) \sigma} \leqslant \frac{M}{\varepsilon} \int_{\mathbb{R}^{2}}|m|^{2} e^{-|m| \sigma} d m \leqslant \frac{17 M}{\varepsilon \sigma^{4}} .
$$

Оценки для производных $\partial \psi / \partial X, \partial \psi / \partial T$ в полосе $D_{\rho_{1}}$ вытекают из леммы 7 . Лемма доказана.

Нам потребуются некоторые утверждения относительно матрицы $C(X, T)$. Выше было установлено, что матрица $C(X, T)$ обладает свойством $\nu$-положительной определенности для вектора частот $\nu=(0,1)$.

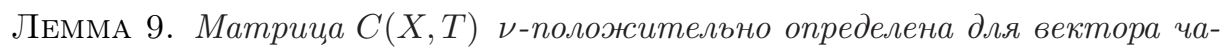
стот $\nu=(\varepsilon, 1)$, если в достаточно мало.

Доказательство сразу вытекает из следующей леммы.

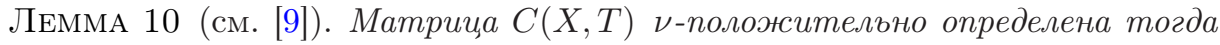
и только тогда, когда существует гладкое семейство положительно определенных матрии, $G(X, T)$ таких, что матрица

$$
\partial_{\nu} G+G C+C^{T} G, \quad \partial_{\nu}=\varepsilon \frac{\partial}{\partial X}+\frac{\partial}{\partial T},
$$

является положительно определенной для любых X и Т. 


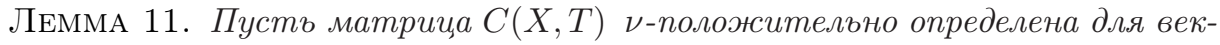
тора частот $\nu=(\varepsilon, 1)$, и пусть вектор $A(X, T)$ периодичен и ограничен в полосе $D_{\rho}$ по норме константой $M$. Тогда существует периодичный вектор $W(X, T)$, удовлетворяющий уравнению

$$
\partial_{\nu} W(X, T)-C(X, T) W(X, T)+A(X, T)=0,
$$

и выполнено неравенство

$$
|W(X, T)|_{\rho} \leqslant \alpha M,
$$

где $\alpha$ - константа, зависящая от матрищы $C$.

ДоказАтельство. Рассмотрим систему

$$
\dot{v}=C(X, T) v, \quad \dot{X}=\varepsilon, \quad \dot{T}=1 .
$$

Пусть $\phi_{t}(X, T)$ - фундаментальная матрица системы, $\phi_{0}(X, T)=I$. Так как $C \nu$-положительно определена, то существуют положительные константы $\lambda, \mu$ такие, что

$$
\left|\phi_{-t}(X, T)\right|_{\rho} \leqslant \mu e^{-\lambda t}, \quad t \geqslant 0 .
$$

Решение уравнения (4.3) имеет вид

$$
W(X, T)=\int_{0}^{+\infty} \phi_{-s}(X, T) A(X+\varepsilon s, T+s) d s .
$$

Учитывая оценку $|A|_{\rho} \leqslant M$, имеем

$$
\left|\int_{0}^{+\infty} \phi_{-s}(X, T) A(X+\varepsilon s, T+s) d s\right|_{\rho} \leqslant \frac{\mu M}{\lambda}=\alpha M .
$$

Лемма доказана.

Аналогичное утверждение верно для уравнения, в котором $W$ и $A$ - матрицы.

ЗАмЕчаниЕ 6. Если вместо $C$ в условиях леммы 11 взять матрицу $C+O(\varepsilon)$, то при достаточно малом $\varepsilon$ изменение оценочного коэффициента $\alpha$ будет порядка $\varepsilon$.

\section{§ 5. КАМ-процедура}

В процессе доказательства будут производиться замены координат. Чтобы не усложнять запись индексами при переменных, будем считать, что каждая замена осуществляет переход от координат без индекса к координатам с индексом 1 :

$$
\left(I, X, Z_{s}, Z_{u}\right) \rightarrow\left(I_{1}, X_{1}, Z_{s 1}, Z_{u 1}\right) .
$$

Функции при степенях переменных $I_{1}, Z_{s 1}, Z_{u 1}$ и функция $F$ будут меняться после каждой замены. Введем у них и у констант, ограничивающих их нормы, индексы, указывающие на порядковый номер замены переменных.

Перепишем гамильтониан (4.1):

$$
H=I+k_{2} I^{2}+\left\langle Z_{s}, C Z_{u}\right\rangle+\Phi_{0}+\Omega_{0}+\Psi_{0}+F_{0},
$$




$$
\begin{gathered}
\Phi_{0}=h_{0}+p_{0} I+q_{0} I^{2}, \\
\Omega_{0}=A_{0} Z_{u}+B_{0} Z_{s}+\left\langle Z_{u}, L_{0} Z_{u}\right\rangle+\left\langle Z_{s}, K_{0} Z_{s}\right\rangle+\left\langle Z_{s}, R_{0} Z_{u}\right\rangle, \\
\Psi_{0}=D_{0} I Z_{u}+Q_{0} I Z_{s}, \quad F_{0}\left(I, X, Z_{s}, Z_{u}, T, \varepsilon\right)=O_{3}\left(I, Z_{s}, Z_{u}\right), \\
C=C(X, T),
\end{gathered}
$$

симплектическая структура $\omega$ имеет вид $\frac{1}{\varepsilon} d I \wedge d X+d Z_{s} \wedge d Z_{u}$.

В области $\Delta_{\rho_{0}}$ справедливы оценки

$$
\begin{array}{cccc}
\left|h_{0}\right| \leqslant M_{0}, & \left|p_{0}\right| \leqslant M_{0}, \quad\left|q_{0}\right| \leqslant M_{0}, \quad\left|A_{0}\right| \leqslant M_{0}, \quad\left|B_{0}\right| \leqslant M_{0}, \\
\left|D_{0}\right| \leqslant M_{0}, & \left|Q_{0}\right| \leqslant M_{0}, \quad\left|K_{0}\right| \leqslant M_{0}, \quad\left|L_{0}\right| \leqslant M_{0}, \quad\left|R_{0}\right| \leqslant M_{0}, \\
\left|F_{0}\right| \leqslant M_{0}^{f} \sum_{\substack{i+j+k=3 \\
i, j, k \geqslant 0}}|I|^{i}\left|Z_{s}\right|^{j}\left|Z_{u}\right|^{k} .
\end{array}
$$

Вообще говоря, $\left\langle p_{0}(X, T)\right\rangle \neq 0$, т.е. частота по $X$ не равна 1. Проведем предварительную замену, сдвигающую частоту:

$$
I=I_{1}+\varepsilon \chi, \quad X=X_{1}, \quad Z_{s}=Z_{s 1}, \quad Z_{u}=Z_{u 1} .
$$

Можно указать такую константу $\chi$, что частота по $X$ будет равна 1 . Так как эта замена предварительная и не входит в KAM-процедуру, не будем указывать оценки и переобозначать коэффициенты. Далее, уже в рамках серии KАМ-замен будет проведена аналогичная замена и показана ее корректность. Будем считать, что $\left\langle h_{0}(X, T)\right\rangle=0$ и $\left\langle q_{0}(X, T)\right\rangle=0$. Последнее соотношение достигается соответствующим изменением $k_{2}$. Приступим к серии KAM-замен.

1. Первая замена имеет своей целью повысить порядок малости $h_{0}$ :

$$
\begin{gathered}
S_{1}\left(I_{1}, X, Z_{s 1}, Z_{u}, T\right)=I_{1} X+Z_{s 1} Z_{u}+\psi_{1}(X, T) \\
I=I_{1}+\varepsilon \frac{\partial \psi_{1}}{\partial X}(X, T), \quad X_{1}=X, \quad Z_{s}=Z_{s 1}, \quad Z_{u 1}=Z_{u}, \\
\frac{\partial S_{1}}{\partial T}=\frac{\partial \psi_{1}}{\partial T}(X, T) .
\end{gathered}
$$

Подставим результат замены в гамильтониан:

$$
H=\left(I_{1}+\varepsilon \frac{\partial \psi_{1}}{\partial X}\right)+k_{2}\left(I_{1}+\varepsilon \frac{\partial \psi_{1}}{\partial X}\right)^{2}+\left\langle Z_{s}, C Z_{u}\right\rangle+\Phi_{0}+\Omega_{0}+\Psi_{0}+F_{0}+\frac{\partial \psi_{1}}{\partial T},
$$

где

$$
\begin{gathered}
\Phi_{0}=h_{0}+p_{0}\left(I_{1}+\varepsilon \frac{\partial \psi_{1}}{\partial X}\right)+q_{0}\left(I_{1}+\varepsilon \frac{\partial \psi_{1}}{\partial X}\right)^{2}, \\
\Omega_{0}=A_{0} Z_{u}+B_{0} Z_{s}+\left\langle Z_{u}, L_{0} Z_{u}\right\rangle+\left\langle Z_{s}, K_{0} Z_{s}\right\rangle+\left\langle Z_{s}, R_{0} Z_{u}\right\rangle \\
\Psi_{0}=D_{0}\left(I_{1}+\varepsilon \frac{\partial \psi_{1}}{\partial X}\right) Z_{u}+Q_{0}\left(I_{1}+\varepsilon \frac{\partial \psi_{1}}{\partial X}\right) Z_{s} \\
F_{0}=F_{0}\left(I_{1}+\varepsilon \frac{\partial \psi_{1}}{\partial X}, X, Z_{s}, Z_{u}, T, \varepsilon\right) .
\end{gathered}
$$


Функцию $\psi_{1}$ найдем из уравнения

$$
\varepsilon \frac{\partial \psi_{1}}{\partial X}(X, T)+\frac{\partial \psi_{1}}{\partial T}(X, T)=-h_{0}(X, T)
$$

Из леммы 8 следует, что в полосе $D_{\rho_{1}}, \rho_{1}=\rho_{0}-2 \sigma$, справедливы оценки

$$
\left|\psi_{1}(X, T)\right| \leqslant \frac{17 M_{0}}{\varepsilon \sigma^{4}}, \quad\left|\frac{\partial \psi_{1}}{\partial X}(X, T)\right| \leqslant \frac{17 M_{0}}{\varepsilon \sigma^{5}} .
$$

ЗАмЕЧАНИЕ 7 . В ходе замены коэффициент $k_{2}$, вообще говоря, изменяется. Далее будет показано, что при достаточно малых $\varepsilon$ совокупные изменения коэффициента $k_{2}$ не превосходят $k_{2} / 2$; для простоты записей оставим прежние обозначения. Также будет показано, что норма функции замены $\psi_{i}$ не превосходит $\sigma$.

При достаточно малых $\varepsilon$ существует положительная, не зависящая от $M_{0}$ и $\varepsilon$ константа $\beta_{1}$ такая, что если обозначить $M_{1}=\beta_{1} M_{0} / \sigma^{5}$ и $M_{1}^{f}=M_{0}^{f}+M_{1}$, то в области $\Delta_{\rho_{1}}$ справедливы оценки

$$
\begin{array}{cccc}
\left|h_{1}\right| \leqslant M_{1}^{2}, & \left|p_{1}\right| \leqslant M_{1}, \quad\left|q_{1}\right| \leqslant M_{1}, \quad\left|A_{1}\right| \leqslant M_{1}, & \left|B_{1}\right| \leqslant M_{1}, \\
\left|D_{1}\right| \leqslant M_{1}, & \left|Q_{1}\right| \leqslant M_{1}, \quad\left|K_{1}\right| \leqslant M_{1}, \quad\left|L_{1}\right| \leqslant M_{1}, \quad\left|R_{1}\right| \leqslant M_{1}, \\
& \left|F_{1}\right| \leqslant M_{1}^{f} \sum_{\substack{i+j+k=3 \\
i, j, k \geqslant 0}}|I|^{i}\left|Z_{s}\right|^{j}\left|Z_{u}\right|^{k} .
\end{array}
$$

Константу $\beta_{1}$ можно указать в явном виде. Достаточно выписать выражения для коэффициентов и слагаемых $h_{1}, \ldots, F_{1}$. Чтобы не усложнять записи, пропустим эти вычисления. В дальнейшем будем указывать оценку только того коэффициента, порядок малости которого повышается.

Заметим, что частота по переменной $X$ изменилась, так как, вообще говоря, $\left\langle p_{1}\left(X_{1}, T\right)\right\rangle \neq 0$; выпишем оценку смещения частоты:

$$
\left|\left\langle p_{1}\left(X_{1}, T\right)\right\rangle\right|_{\rho_{1}}=\left|\left\langle 2 q_{0} \varepsilon \frac{\partial \psi_{1}}{\partial X}+\frac{\partial F_{0}}{\partial I}\left(\varepsilon \frac{\partial \psi_{1}}{\partial X}, X_{1}, 0,0, T\right)\right\rangle\right|_{\rho_{1}} \leqslant M_{1}^{2} .
$$

2. Вторая замена направлена на повышение порядка малости $p_{1}$ :

$$
\begin{gathered}
S_{2}\left(I_{1}, X, Z_{s 1}, Z_{u}, T\right)=I_{1} X+Z_{s 1} Z_{u}+\psi_{2}(X, T) I_{1}, \\
I=I_{1}+\varepsilon \frac{\partial \psi_{2}}{\partial X}(X, T) I_{1}, \quad X_{1}=X+\varepsilon \psi_{2}(X, T), \quad Z_{s}=Z_{s 1}, \quad Z_{u 1}=Z_{u}, \\
\frac{\partial S_{2}}{\partial T}=\frac{\partial \psi_{2}}{\partial T}(X, T) I_{1} .
\end{gathered}
$$

Подставим результат замены в гамильтониан:

$H=\left(1+\varepsilon \frac{\partial \psi_{2}}{\partial X}\right) I_{1}+k_{2}\left(1+\varepsilon \frac{\partial \psi_{2}}{\partial X}\right)^{2} I_{1}^{2}+\left\langle Z_{s}, C Z_{u}\right\rangle+\Phi_{1}+\Omega_{1}+\Psi_{1}+F_{1}+\frac{\partial \psi_{2}}{\partial T} I_{1}$, 
где

$$
\begin{gathered}
\Phi_{1}=h_{1}+p_{1}\left(1+\varepsilon \frac{\partial \psi_{2}}{\partial X}\right) I_{1}+q_{1}\left(1+\varepsilon \frac{\partial \psi_{2}}{\partial X}\right)^{2} I_{1}^{2}, \\
\Omega_{1}=A_{1} Z_{u}+B_{1} Z_{s}+\left\langle Z_{u}, L_{1} Z_{u}\right\rangle+\left\langle Z_{s}, K Z_{s}\right\rangle+\left\langle Z_{s}, R_{1} Z_{u}\right\rangle \\
\Psi_{1}=D_{1}\left(1+\varepsilon \frac{\partial \psi_{2}}{\partial X}\right) I_{1} Z_{u}+Q_{1}\left(1+\varepsilon \frac{\partial \psi_{2}}{\partial X}\right) I_{1} Z_{s} \\
F_{1}=F_{1}\left(\left(1+\varepsilon \frac{\partial \psi_{2}}{\partial X}\right) I_{1}, X, Z_{s}, Z_{u}, T, \varepsilon\right) .
\end{gathered}
$$

Функцию $\psi_{2}$ найдем из уравнения

$$
\varepsilon \frac{\partial \psi_{2}}{\partial X}(X, T)+\frac{\partial \psi_{2}}{\partial T}(X, T)=-p_{1}(X, T)+\left\langle p_{1}(X, T)\right\rangle .
$$

Из леммы 8 следует, что в области $D_{\rho_{2}^{\prime}}, \rho_{2}^{\prime}=\rho_{1}-2 \sigma$, справедливы оценки

$$
\left|\psi_{2}(X, T)\right| \leqslant \frac{17 M_{1}}{\varepsilon \sigma^{4}}, \quad\left|\frac{\partial \psi_{1}}{\partial X}(X, T)\right| \leqslant \frac{17 M_{1}}{\varepsilon \sigma^{5}} .
$$

В отличие от первой замены, преобразование координаты $X$ не является тождественным. Разрешая уравнение относительно $X$ в полосе $D_{\rho_{2}}, \rho_{2}=\rho_{2}^{\prime}-\sigma$, имеем

$$
X=X_{1}+\varepsilon \psi_{2}^{*}\left(X_{1}, T\right), \quad\left|\varepsilon \psi_{2}^{*}\left(X_{1}, T\right)\right| \leqslant 2 \frac{17 M_{1}}{\sigma^{4}} .
$$

Положив $M_{2}=\beta_{2} M_{1} / \sigma^{5}$ и $M_{2}^{f}=M_{1}^{f}+M_{2}$, в области $\Delta_{\rho_{2}}$ имеем оценку $\left|p_{2}\right| \leqslant M_{2}^{2}$.

3. Третья замена повышает порядок малости $q_{2}$ :

$$
\begin{gathered}
S_{3}\left(I_{1}, X, Z_{s 1}, Z_{u}, T\right)=I_{1} X+Z_{s 1} Z_{u}+\psi_{3}(X, T) I_{1}^{2} \\
I=I_{1}+\varepsilon \frac{\partial \psi_{3}}{\partial X}(X, T) I_{1}^{2}, \quad X_{1}=X+2 \varepsilon \psi_{3}(X, T) I_{1}, \quad Z_{s}=Z_{s 1}, \quad Z_{u 1}=Z_{u}, \\
\frac{\partial S_{3}}{\partial T}=\frac{\partial \psi_{3}}{\partial T}(X, T) I_{1}^{2} .
\end{gathered}
$$

Подставим результат замены в гамильтониан:

$$
\begin{aligned}
H=( & \left.I_{1}+\varepsilon \frac{\partial \psi_{3}}{\partial X} I_{1}^{2}\right)+k_{2}\left(I_{1}+\varepsilon \frac{\partial \psi_{3}}{\partial X} I_{1}^{2}\right)^{2}+\left\langle Z_{s}, C Z_{u}\right\rangle \\
& +\Phi_{2}+\Omega_{2}+\Psi_{2}+F_{2}+\frac{\partial \psi_{3}}{\partial T} I_{1}^{2}
\end{aligned}
$$

где

$$
\begin{gathered}
\Phi_{2}=h_{2}+p_{2}\left(I_{1}+\varepsilon \frac{\partial \psi_{3}}{\partial X} I_{1}^{2}\right)+q_{2}\left(I_{1}+\varepsilon \frac{\partial \psi_{3}}{\partial X} I_{1}^{2}\right)^{2}, \\
\Omega_{2}=A_{2} Z_{u}+B_{2} Z_{s}+\left\langle Z_{u}, L_{2} Z_{u}\right\rangle+\left\langle Z_{s}, K_{2} Z_{s}\right\rangle+\left\langle Z_{s}, R_{2} Z_{u}\right\rangle, \\
\Psi_{2}=D_{2}\left(I_{1}+\varepsilon \frac{\partial \psi_{3}}{\partial X} I_{1}^{2}\right) Z_{u}+Q_{2}\left(I_{1}+\varepsilon \frac{\partial \psi_{3}}{\partial X} I_{1}^{2}\right) Z_{s}, \\
F_{2}=F_{2}\left(I_{1}+\varepsilon \frac{\partial \psi_{3}}{\partial X} I_{1}^{2}, X, Z_{s}, Z_{u}, T, \varepsilon\right) .
\end{gathered}
$$


Функцию $\psi_{3}$ найдем из уравнения

$$
\varepsilon \frac{\partial \psi_{3}}{\partial X}(X, T)+\frac{\partial \psi_{3}}{\partial T}(X, T)=-q_{2}(X, T) .
$$

Из леммы 8 следует, что в полосе $D_{\rho_{3}^{\prime}}, \rho_{3}^{\prime}=\rho_{2}-2 \sigma$, справедливы оценки

$$
\left|\psi_{3}(X, T)\right| \leqslant \frac{17 M_{2}}{\varepsilon \sigma^{4}}, \quad\left|\frac{\partial \psi_{3}}{\partial X}(X, T)\right| \leqslant \frac{17 M_{2}}{\varepsilon \sigma^{5}} .
$$

Как и в случае второй замены, преобразование $X$ не тождественно, в области $\Delta_{\rho_{3}}, \rho_{3}=\rho_{3}^{\prime}-\sigma$, имеем

$$
X=X_{1}+2 \varepsilon \psi_{3}^{*}\left(X_{1}, T, I_{1}\right), \quad\left|2 \varepsilon \psi_{3}^{*}\left(X_{1}, T, I_{1}\right)\right| \leqslant 4 \frac{17 M_{2}}{\sigma^{4}} .
$$

Как и ранее, введем константу $\beta_{3}$ и обозначим $M_{3}=\beta_{3} M_{2} / \sigma^{5}, M_{3}^{f}=M_{2}^{f}+M_{3}$. В области $\Delta_{\rho_{3}}$ справедливо неравенство $\left|q_{3}\right| \leqslant M_{3}^{2}$.

4. Четвертая замена повысит порядок малости $A_{3}$ :

$$
\begin{aligned}
S_{4}\left(I_{1}, X, Z_{s 1}, Z_{u}, T\right) & =I_{1} X+Z_{s 1} Z_{u}+\psi_{4}(X, T) Z_{s 1}, \\
I=I_{1}+\varepsilon \frac{\partial \psi_{4}}{\partial X}(X, T) Z_{s 1}, \quad X_{1} & =X, \quad Z_{s}=Z_{s 1}, \quad Z_{u 1}=Z_{u}+\psi_{4}(X, T), \\
\frac{\partial S_{4}}{\partial T} & =\frac{\partial \psi_{4}}{\partial T}(X, T) Z_{s 1} .
\end{aligned}
$$

Подставим результат замены в гамильтониан:

$$
\begin{aligned}
H=( & \left.I_{1}+\varepsilon \frac{\partial \psi_{4}}{\partial X} Z_{s 1}\right)+k_{2}\left(I_{1}+\varepsilon \frac{\partial \psi_{4}}{\partial X} Z_{s 1}\right)^{2}+\left\langle Z_{s}, C\left(Z_{u 1}-\psi_{4}\right)\right\rangle \\
& +\Phi_{3}+\Omega_{3}+\Psi_{3}+F_{3}+\frac{\partial \psi_{4}}{\partial T} Z_{s 1}
\end{aligned}
$$

где

$$
\begin{gathered}
\Phi_{3}=h_{3}+p_{3}\left(I_{1}+\varepsilon \frac{\partial \psi_{4}}{\partial X} Z_{s 1}\right)+q_{3}\left(I_{1}+\varepsilon \frac{\partial \psi_{4}}{\partial X} Z_{s 1}\right)^{2}, \\
\Omega_{3}=A_{3}\left(Z_{u 1}-\psi_{4}\right)+B_{3} Z_{s}+\left\langle\left(Z_{u 1}-\psi_{4}\right), L_{3}\left(Z_{u 1}-\psi_{4}\right)\right\rangle \\
+\left\langle Z_{s}, K_{3} Z_{s}\right\rangle+\left\langle Z_{s}, R_{3}\left(Z_{u 1}-\psi_{4}\right)\right\rangle \\
\Psi_{3}=D_{3}\left(I_{1}+\varepsilon \frac{\partial \psi_{4}}{\partial X} Z_{s 1}\right)\left(Z_{u 1}-\psi_{4}\right)+Q_{3}\left(I_{1}+\varepsilon \frac{\partial \psi_{4}}{\partial X} Z_{s 1}\right) Z_{s}, \\
F_{3}=F_{3}\left(I_{1}+\varepsilon \frac{\partial \psi_{4}}{\partial X} Z_{s 1}, X, Z_{s}, Z_{u 1}-\psi_{4}, T, \varepsilon\right) .
\end{gathered}
$$

Функцию $\psi_{4}$ найдем из уравнения

$$
\varepsilon \frac{\partial \psi_{4}}{\partial X}(X, T)+\frac{\partial \psi_{4}}{\partial T}(X, T)-C(X, T) \psi_{4}(X, T)=-A_{3}(X, T) .
$$

Согласно лемме 11 существует решение этого уравнения $\psi_{4}(X, T)$ и в полосе $D_{\rho_{4}}, \rho_{4}=\rho_{3}-\sigma$, справедливы оценки

$$
\left|\psi_{4}(X, T)\right| \leqslant \alpha M_{3}, \quad\left|\frac{\partial \psi_{4}}{\partial X}(X, T)\right| \leqslant \frac{\alpha}{\sigma} M_{3} .
$$


Введем обозначения $M_{4}=\beta_{4} M_{3} / \sigma$ и $M_{4}^{f}=M_{3}^{f}+M_{4}$. В области $\Delta_{\rho_{4}}$ справедлива оценка $\left|A_{4}\right| \leqslant M_{4}^{2}$.

5. Пятая замена, повышающая порядок малости $B_{4}$, аналогична предыдущей:

$$
\begin{aligned}
S_{5}\left(I_{1}, X, Z_{s 1}, Z_{u}, T\right) & =I_{1} X+Z_{s 1} Z_{u}+\psi_{5}(X, T) Z_{u} \\
I=I_{1}+\varepsilon \frac{\partial \psi_{5}}{\partial X}(X, T) Z_{u}, \quad X_{1} & =X, \quad Z_{s}=Z_{s 1}+\psi_{5}(X, T), \quad Z_{u 1}=Z_{u}, \\
\frac{\partial S_{5}}{\partial T} & =\frac{\partial \psi_{5}}{\partial T}(X, T) Z_{u} .
\end{aligned}
$$

Функцию $\psi_{5}$ найдем из уравнения

$$
\varepsilon \frac{\partial \psi_{5}}{\partial X}(X, T)+\frac{\partial \psi_{5}}{\partial T}(X, T)+\psi_{5}(X, T) C(X, T)=-B_{4}(X, T) .
$$

Оценки для решения этого, а также схожих гомологических уравнений в последующих двух заменах аналогичны оценкам из леммы 11 . В области $\Delta_{\rho_{5}}$, $\rho_{5}=\rho_{4}-\sigma$, имеем $\left|B_{5}\right| \leqslant M_{5}^{2}$.

6. Шестая замена направлена на повышение порядка малости $K_{5}$ :

$$
\begin{gathered}
S_{6}\left(I_{1}, X, Z_{s 1}, Z_{u}, T\right)=I_{1} X+Z_{s 1} Z_{u}+\left\langle Z_{s 1}, \psi_{6}(X, T) Z_{s 1}\right\rangle, \\
I=I_{1}+\varepsilon\left\langle Z_{s 1}, \frac{\partial \psi_{6}}{\partial X}(X, T) Z_{s 1}\right\rangle, \quad X_{1}=X \\
Z_{s}=Z_{s 1}, \quad Z_{u 1}=Z_{u}+\psi_{6}(X, T) Z_{s 1}+\psi_{6}^{T}(X, T) Z_{s 1}, \\
\frac{\partial S_{6}}{\partial T}=\left\langle Z_{s 1}, \frac{\partial \psi_{6}}{\partial T}(X, T) Z_{s 1}\right\rangle .
\end{gathered}
$$

Подставим результат замены в гамильтониан:

$$
\begin{aligned}
H=( & \left.I_{1}+\varepsilon\left\langle Z_{s 1}, \frac{\partial \psi_{6}}{\partial X} Z_{s 1}\right\rangle\right)+k_{2}\left(I_{1}+\varepsilon\left\langle Z_{s 1}, \frac{\partial \psi_{6}}{\partial X} Z_{s 1}\right\rangle\right)^{2} \\
& +\left\langle Z_{s}, C\left(Z_{u 1}-\psi_{6} Z_{s 1}-\psi_{6}^{T} Z_{s 1}\right)\right\rangle+\Phi_{5}+\Omega_{5}+\Psi_{5}+F_{5}+\left\langle Z_{s 1}, \frac{\partial \psi_{6}}{\partial T} Z_{s 1}\right\rangle,
\end{aligned}
$$

где

$$
\begin{aligned}
& \Phi_{5}=h_{5}+ p_{5}\left(I_{1}+\varepsilon\left\langle Z_{s 1}, \frac{\partial \psi_{6}}{\partial X} Z_{s 1}\right\rangle\right)+q_{5}\left(I_{1}+\varepsilon\left\langle Z_{s 1}, \frac{\partial \psi_{6}}{\partial X} Z_{s 1}\right\rangle\right)^{2}, \\
& \Omega_{5}=A_{5}\left(Z_{u 1}-\psi_{6} Z_{s 1}-\psi_{6}^{T} Z_{s 1}\right)+B_{5} Z_{s} \\
&+\left\langle\left(Z_{u 1}-\psi_{6} Z_{s 1}-\psi_{6}^{T} Z_{s 1}\right), L_{5}\left(Z_{u 1}-\psi_{6} Z_{s 1}-\psi_{6}^{T} Z_{s 1}\right)\right\rangle \\
&+\left\langle Z_{s}, K_{5} Z_{s}\right\rangle+\left\langle Z_{s}, R_{5}\left(Z_{u 1}-\psi_{6} Z_{s 1}-\psi_{6}^{T} Z_{s 1}\right)\right\rangle \\
& \Psi_{5}= D_{5}\left(I_{1}+\varepsilon\left\langle Z_{s 1}, \frac{\partial \psi_{6}}{\partial X} Z_{s 1}\right\rangle\right)\left(Z_{u 1}-\psi_{6} Z_{s 1}-\psi_{6}^{T} Z_{s 1}\right) \\
&+Q_{5}\left(I_{1}+\varepsilon\left\langle Z_{s 1}, \frac{\partial \psi_{6}}{\partial X} Z_{s 1}\right\rangle\right) Z_{s}, \\
& F_{5}=F_{5}\left(I_{1}+\varepsilon\left\langle Z_{s 1}, \frac{\partial \psi_{6}}{\partial X} Z_{s 1}\right\rangle, X, Z_{s}, Z_{u 1}-\psi_{6} Z_{s 1}-\psi_{6}^{T} Z_{s 1}, T, \varepsilon\right) .
\end{aligned}
$$


Функцию $\psi_{6}$ найдем из уравнения

$$
\varepsilon \frac{\partial \psi_{6}}{\partial X}(X, T)+\frac{\partial \psi_{6}}{\partial T}-C(X, T) \psi_{6}(X, T)-\psi_{6}(X, T) C^{T}(X, T)=-K_{5}(X, T) .
$$

В полосе $D_{\rho_{6}}, \rho_{6}=\rho_{5}-\sigma$, имеем оценки

$$
\left|\psi_{6}(X, T)\right| \leqslant \alpha M_{5}, \quad\left|\frac{\partial \psi_{6}}{\partial X}(X, T)\right| \leqslant \frac{\alpha}{\sigma} M_{5}
$$

Как и выше, введем константы $M_{6}=\beta_{6} M_{5} / \sigma$ и $M_{6}^{f}=M_{5}^{f}+M_{6}$. В области $\Delta_{\rho_{6}}$ выполнена оценка $\left|K_{6}\right| \leqslant M_{6}^{2}$.

7. Седьмая замена повысит порядок малости $L_{6}$ :

$$
\begin{gathered}
S_{7}\left(I_{1}, X, Z_{u 1}, Z_{s}, T\right)=I_{1} X+Z_{s} Z_{u 1}+\left\langle Z_{u}, \psi_{7}(X, T) Z_{u}\right\rangle \\
I=I_{1}+\varepsilon\left\langle Z_{u}, \frac{\partial \psi_{7}}{\partial X}(X, T) Z_{u}\right\rangle, \quad X_{1}=X \\
Z_{s}=Z_{s 1}+\psi_{7}(X, T) Z_{u}+\psi_{7}^{T}(X, T) Z_{u}, \quad Z_{u 1}=Z_{u}, \\
\frac{\partial S_{7}}{\partial T}=\left\langle Z_{u}, \frac{\partial \psi_{7}}{\partial T}(X, T) Z_{u}\right\rangle
\end{gathered}
$$

Функцию $\psi_{7}$ найдем из уравнения

$$
\varepsilon \frac{\partial \psi_{7}}{\partial X}(X, T)+\frac{\partial \psi_{7}}{\partial T}+\psi_{7}(X, T) C(X, T)+C^{T}(X, T) \psi_{7}(X, T)=-L_{5}(X, T) .
$$

Замена аналогична предыдущей, в ходе нее в области $\Delta_{\rho_{7}}, \rho_{7}=\rho_{6}-\sigma$, коэффициент $L_{7}$ повысит порядок малости: $\left|L_{7}\right| \leqslant M_{7}^{2}$.

8. Восьмая замена повышает порядок малости $Q_{7}$ :

$$
\begin{gathered}
S_{8}\left(I_{1}, X, Z_{s 1}, Z_{u}, T\right)=I_{1} X+Z_{s 1} Z_{u}+\psi_{8}(X, T) I_{1} Z_{s 1}, \\
I=I_{1}+\varepsilon \frac{\partial \psi_{8}}{\partial X}(X, T) I_{1} Z_{s 1}, \quad X_{1}=X+\varepsilon \psi_{8}(X, T) Z_{s 1} \\
Z_{s}=Z_{s 1}, \quad Z_{u 1}=Z_{u}+\psi_{8}(X, T) I_{1}, \\
\frac{\partial S_{8}}{\partial T}=\frac{\partial \psi_{8}}{\partial T}(X, T) I_{1} Z_{s 1} .
\end{gathered}
$$

Подставим результат замены в гамильтониан:

$$
\begin{aligned}
H=( & \left.I_{1}+\varepsilon \frac{\partial \psi_{8}}{\partial X} I_{1} Z_{s 1}\right)+k_{2}\left(I_{1}+\varepsilon \frac{\partial \psi_{8}}{\partial X} I_{1} Z_{s 1}\right)^{2}+\left\langle Z_{s}, C\left(Z_{u 1}-\psi_{8} I_{1}\right)\right\rangle \\
& +\Phi_{7}+\Omega_{7}+\Psi_{7}+F_{7}+\frac{\partial \psi_{8}}{\partial T} I_{1} Z_{s 1}
\end{aligned}
$$

где

$$
\begin{gathered}
\Phi_{7}=h_{7}+p_{7}\left(I_{1}+\varepsilon \frac{\partial \psi_{8}}{\partial X} I_{1} Z_{s 1}\right)+q_{7}\left(I_{1}+\varepsilon \frac{\partial \psi_{8}}{\partial X} I_{1} Z_{s 1}\right)^{2}, \\
\Omega_{7}=A_{7}\left(Z_{u 1}-\psi_{8} I_{1}\right)+B_{7} Z_{s}+\left\langle\left(Z_{u 1}-\psi_{8} I_{1}\right), L_{7}\left(Z_{u 1}-\psi_{8} I_{1}\right)\right\rangle \\
+\left\langle Z_{s}, K_{7} Z_{s}\right\rangle+\left\langle Z_{s}, R_{7}\left(Z_{u 1}-\psi_{8} I_{1}\right)\right\rangle
\end{gathered}
$$




$$
\begin{gathered}
\Psi_{7}=D_{7}\left(I_{1}+\varepsilon \frac{\partial \psi_{8}}{\partial X} I_{1} Z_{s 1}\right)\left(Z_{u 1}-\psi_{8} I_{1}\right)+Q_{7}\left(I_{1}+\varepsilon \frac{\partial \psi_{8}}{\partial X} I_{1} Z_{s 1}\right) Z_{s} \\
F_{7}=F_{7}\left(I_{1}+\varepsilon \frac{\partial \psi_{8}}{\partial X} I_{1} Z_{s 1}, X, Z_{s}, Z_{u 1}-\psi_{8} I_{1}, T, \varepsilon\right)
\end{gathered}
$$

Функцию $\psi_{8}$ найдем из уравнения

$$
\varepsilon \frac{\partial \psi_{8}}{\partial X}(X, T)+\frac{\partial \psi_{8}}{\partial T}-C(X, T) \psi_{8}(X, T)=-Q_{7}(X, T) .
$$

Согласно лемме 11 существует решение этого уравнения $\psi_{8}(X, T)$. В полосе $D_{\rho_{8}^{\prime}}, \rho_{8}^{\prime}=\rho_{7}-\sigma$, справедливы оценки

$$
\left|\psi_{8}(X, T)\right| \leqslant \alpha M_{7}, \quad\left|\frac{\partial \psi_{8}}{\partial X}(X, T)\right| \leqslant \frac{\alpha}{\sigma} M_{7} .
$$

Преобразование $X$ не тождественно, в области $\Delta_{\rho_{8}}, \rho_{8}=\rho_{8}^{\prime}-\sigma$, имеем

$$
X=X_{1}+\varepsilon \psi_{8}^{*}\left(X_{1}, T, Z_{s 1}\right), \quad\left|\varepsilon \psi_{8}^{*}\left(X_{1}, T, Z_{s 1}\right)\right| \leqslant 2 \varepsilon \alpha M_{7} .
$$

Обозначив $M_{8}=\beta_{8} M_{7} / \sigma$ и $M_{8}^{f}=M_{7}^{f}+M_{8}$, получим оценку $\left|L_{8}\right| \leqslant M_{8}^{2}$.

9. Девятая замена повысит порядок малости $D_{9}$ :

$$
\begin{gathered}
S_{9}\left(I_{1}, X, Z_{s 1}, Z_{u}, T\right)=I_{1} X+Z_{s 1} Z_{u}+\psi_{9}(X, T) I_{1} Z_{u} \\
I=I_{1}+\varepsilon \frac{\partial \psi_{9}}{\partial X}(X, T) I_{1} Z_{u}, \quad X_{1}=X+\varepsilon \psi_{9}(X, T) Z_{u} \\
Z_{s}=Z_{s 1}+\psi_{9}(X, T) I_{1}, \quad Z_{u 1}=Z_{u} \\
\frac{\partial S_{9}}{\partial T}=\frac{\partial \psi_{9}}{\partial T}(X, T) I_{1} Z_{u} .
\end{gathered}
$$

Функцию $\psi_{9}$ найдем из уравнения

$$
\varepsilon \frac{\partial \psi_{9}}{\partial X}(X, T)+\frac{\partial \psi_{9}}{\partial T}+\psi_{9}(X, T) C(X, T)=-D_{9}(X, T) .
$$

Замена аналогична предыдущей. В ходе нее в области $\Delta_{\rho_{9}}, \rho_{9}=\rho_{8}-2 \sigma$, коэффициент $D_{9}$ будет иметь оценку $\left|D_{9}\right| \leqslant M_{9}^{2}$.

10. Так как, вообще говоря, $\left\langle p_{9}(X, T)\right\rangle \neq 0$, то необходимо провести замену, сдвигающую частоту по переменной $X$ :

$$
I=I_{1}+\varepsilon \chi, \quad X_{1}=X, \quad Z_{s}=Z_{s 1}, \quad Z_{u 1}=Z_{u} .
$$

Подставим результат замены в гамильтониан:

$$
H=\left(I_{1}+\varepsilon \chi\right)+k_{2}\left(I_{1}+\varepsilon \chi\right)^{2}+\left\langle Z_{s}, C Z_{u}\right\rangle+\Phi_{9}+\Omega_{9}+\Psi_{9}+F_{9},
$$

где

$$
\begin{gathered}
\Phi_{9}=h_{9}+p_{9}\left(I_{1}+\varepsilon \chi\right)+q_{9}\left(I_{1}+\varepsilon \chi\right)^{2} \\
\Omega_{9}=A_{9} Z_{u}+B_{9} Z_{s}+\left\langle Z_{u}, L_{9} Z_{u}\right\rangle+\left\langle Z_{s}, K_{9} Z_{s}\right\rangle+\left\langle Z_{s}, R_{9} Z_{u}\right\rangle \\
\Psi_{9}=D_{9}\left(I_{1}+\varepsilon \chi\right) Z_{u}+Q_{9}\left(I_{1}+\varepsilon \chi\right) Z_{s}, \quad F_{9}=F_{9}\left(I_{1}+\varepsilon \chi, X, Z_{s}, Z_{u}, T, \varepsilon\right) .
\end{gathered}
$$


Константу $\chi$ найдем из уравнения

$$
2 k_{2} \varepsilon \chi+\left\langle\frac{\partial F_{9}}{\partial I}(\varepsilon \chi, X, 0,0, T, \varepsilon)\right\rangle=-\left\langle p_{9}(X, T)\right\rangle .
$$

Уравнение (5.1) имеет решение, удовлетворяющее неравенству

$$
|\varepsilon \chi| \leqslant \frac{M_{9}^{2}}{\left|k_{2}\right|} .
$$

Обозначив $M_{10}=\beta_{10} M_{9} / \sigma$ и $M_{10}^{f}=M_{9}^{f}+M_{10}$, выпишем окончательные оценки на функции в области $\Delta_{\rho_{10}}, \rho_{10}=\rho_{9}-\sigma$ :

$$
\begin{gathered}
\left|h_{10}\right| \leqslant M_{10}^{2}, \quad\left|p_{10}\right| \leqslant M_{10}^{2}, \quad\left|q_{10}\right| \leqslant M_{10}^{2}, \quad\left|A_{10}\right| \leqslant M_{10}^{2}, \quad\left|B_{10}\right| \leqslant M_{10}^{2}, \\
\left|D_{10}\right| \leqslant M_{10}^{2}, \quad\left|Q_{10}\right| \leqslant M_{10}^{2}, \quad\left|K_{10}\right| \leqslant M_{10}^{2}, \quad\left|L_{10}\right| \leqslant M_{10}^{2}, \quad\left|R_{10}\right| \leqslant M_{10}, \\
\left|F_{10}\right| \leqslant M_{10}^{f} \sum_{\substack{i+j+k=3 \\
i, j, k \geqslant 0}}|I|^{i}\left|Z_{s}\right|^{j}\left|Z_{u}\right|^{k} .
\end{gathered}
$$

Сделаем заключительное преобразование. Пусть

$$
\widetilde{C}(X, T)=C(X, T)+R_{10}(X, T) .
$$

Учтем, что $\nu$-положительная определенность сохраняется при малых изменениях матрицы $C$. Перепишем гамильтониан:

$$
H=I+k_{2} I^{2}+\left\langle Z_{s}, \widetilde{C} Z_{u}\right\rangle+\Phi_{10}+\Omega_{10}+\Psi_{10}+F_{10},
$$

где

$$
\begin{gathered}
\Phi_{10}=h_{10}+p_{10} I+q_{10} I^{2} \\
\Omega_{10}=A_{10} Z_{u}+B_{10} Z_{s}+\left\langle Z_{u}, L_{10} Z_{u}\right\rangle+\left\langle Z_{s}, K_{10} Z_{s}\right\rangle, \\
\Psi_{10}=D_{10} I Z_{u}+Q_{10} I Z_{s} .
\end{gathered}
$$

В области $\Delta_{\rho_{10}}$ коэффициенты $h_{10}, \ldots, Q_{10}$ ограничены по норме константой $M_{10}^{2}$. Выразим $M_{10}$ и $M_{10}^{f}$ через начальные оценки:

$$
M_{10}=\prod_{i=1}^{10} \beta_{i} \frac{M_{0}}{\sigma^{22}}=\widetilde{\beta} \frac{M_{0}}{\sigma^{22}}, \quad M_{10}^{f} \leqslant M_{0}^{f}+10 M_{10} .
$$

Представленные 10 замен являются первым шагом КАМ-процедуры. Сделаем индуктивный переход к произвольному шагу и покажем сходимость преобразований при малых $\varepsilon$.

Зададим закон сужения комплексной области. Пусть на шаге $i$ величина уменьшения рассматриваемой области задается формулой $\sigma_{i}=\frac{1}{44} \rho_{0} 2^{-i}$. Оценку порядка малости членов гамильтониана на $i$-м шаге будем обозначать $\widetilde{M}_{i}$, оценку на функцию $F$ обозначим $\widetilde{M}_{i}^{f}$. Справедливы соотношения

$$
\begin{gathered}
\widetilde{M}_{i+1}=\frac{\widetilde{\beta}^{2}}{\sigma_{i}^{44}} \widetilde{M}_{i}^{2}=44^{44} \widetilde{\beta}^{2} \frac{2^{44 i}}{\rho_{0}^{44}} \widetilde{M}_{i}^{2}, \quad \widetilde{M}_{i+1}^{f} \leqslant \widetilde{M}_{i}^{f}+10 \sqrt{\widetilde{M}_{i+1}}, \\
\widetilde{M}_{1}=M_{0}, \quad \widetilde{M}_{1}^{f}=M_{0}^{f} .
\end{gathered}
$$

Докажем вспомогательную лемму. Рассмотрим рекуррентную последовательность $b_{n+1}=\xi \gamma^{n}\left(b_{n}\right)^{2}$, где $\xi, \gamma$ - положительные константы. 
ЛЕмма 12. (n+1)-й член рекуррентной последовательности $\left\{b_{n}\right\}$ выражается через первый по формуле

$$
\begin{gathered}
b_{n+1}=\xi^{\left(2^{0}+2^{1}+2^{2}+\cdots+2^{n-1}\right)} \gamma^{\left(2^{0} n+2^{1}(n-1)+2^{2}(n-2)+\cdots+2^{n-1}\right)} b_{1}^{2^{n}}, \\
b_{n+1} \leqslant \xi^{2^{n}-1} \gamma^{2^{n+1}} b_{1}^{2^{n}} .
\end{gathered}
$$

ДокАЗАТЕльство. Воспользуемся методом математической индукции. При $n=1$ имеем $b_{2}=\xi \gamma\left(b_{1}\right)^{2}$. Предположим, что формула верна для некоторого номера $n$. Подставим равенство в выражение для номера $n+1$ :

$$
\begin{aligned}
b_{n+2} & =\xi \gamma^{n+1}\left(b_{n+1}\right)^{2}=\xi \gamma^{n+1}\left(\xi^{\left(2^{0}+2^{1}+2^{2}+\cdots+2^{n-1}\right)} \gamma^{\left(2^{0} n+2^{1}(n-1)+\cdots+2^{n-1}\right)} b_{1}^{2^{n}}\right)^{2} \\
& =\xi^{\left(2^{0}+2^{1}+2^{2}+\cdots+2^{n}\right)} \gamma^{\left(2^{0}(n+1)+2^{1} n+\cdots+2^{n}\right)} b_{1}^{2^{n+1}} .
\end{aligned}
$$

Мы получили требуемую формулу. Преобразуем ряды:

$$
\begin{gathered}
2^{0}+2^{1}+2^{2}+\cdots+2^{n-1}=2^{n}-1, \\
2^{0} n+2^{1}(n-1)+2^{2}(n-2)+\cdots+2^{n-1} \\
\quad=2^{n}\left(\frac{n}{2^{n}}+\frac{n-1}{2^{(n-1)}}+\cdots+\frac{1}{2}\right)<2^{n+1} .
\end{gathered}
$$

Последние оценки дают неравенство для $b_{n+1}$. Лемма доказана.

СлеДСТвИЕ. Для любых положительных $\xi$ и можно указатъ такое $b_{1}$, что последовательность $\left\{b_{n}\right\}$ будет сходиться $\kappa$ нулю. Выбором малого $b_{1}$ можно получить сходящуюся последовательность, каждый член которой менъше соответствующего ему члена любой наперед заданной геометрической прогрессии. В частности, прогрессии $\sigma_{n}$.

В нашей задаче $\xi=44^{44} \widetilde{\beta}^{2} \rho_{0}^{-44}, \gamma=2^{44}$. Справедливость замечания 7 вытекает из этого следствия. Окончательно заключаем, что начиная с некоторого достаточно малого начального значения $\widetilde{M}_{1}$ последовательность $\left\{\widetilde{M}_{i}\right\}$ будет сходиться к нулю, а последовательность $\left\{\widetilde{M}_{i}^{f}\right\}$ не будет превосходить $2 \widetilde{M}_{1}^{f}$. Соответственно существует $\varepsilon_{0}>0$ такое, что для любого $\varepsilon \in\left(0, \varepsilon_{0}\right)$, для которого вектор частот $(\varepsilon, 1)$ диофантов, последовательность КАМ-преобразований сходится. Осуществив предельный переход, в области $\Delta_{\rho_{0} / 2}$ получим систему координат $\left(I_{\infty}, X_{\infty}, Z_{s \infty}, Z_{u \infty}, T\right)$, в которых функция Гамильтона имеет вид

$$
H=I_{\infty}+k_{2} I_{\infty}^{2}+\left\langle Z_{s \infty}, C_{\infty}\left(X_{\infty}, T\right) Z_{u \infty}\right\rangle+F_{\infty}\left(I_{\infty}, X_{\infty}, Z_{s \infty}, Z_{u \infty}, T, \varepsilon\right),
$$

где $F_{\infty}=O_{3}\left(I_{\infty}, Z_{s_{\infty}}, Z_{u_{\infty}}\right)$, матрица $C_{\infty} \nu$-положительно определена и $\nu=$ $(\varepsilon, 1)$. Заметим, что справедливо соотношение $I_{\infty}=I+O(\varepsilon)$, где $I$ - координата до проведения КАМ-преобразований. Остается перейти к стандартной симплектической структуре.

Теорема 1 полностью доказана.

\section{Список литературы}

[1] В.И. Арнольд, "О поведении адиабатического инварианта при медленном периодическом изменении функции Гамильтона", Докл. АН СCCP, 142:4 (1962), 758-761; англ. пер.: V.I. Arnol'd, "On the behavior of an adiabatic invariant under slow periodic variation of the Hamiltonian", Soviet Math. Dokl., 3 (1962), 136-140. 
[2] S. M. Graff, "On the conservation of hyperbolic invariant tori for Hamiltonian systems", J. Differential Equations, 15 (1974), 1-69.

[3] Ю. Н. Бибиков, "Усиление одной теоремы Мозера", Докл. АН СССР, 213:4 (1973), 766-769; англ. пер.: Yu. N. Bibikov, "A sharpening of a theorem of Moser", Soviet Math. Dokl., 14 (1973), 1769-1773.

[4] Г.Н. Пифтанкин, "Скорость диффузии в задаче Мезера", Докл. РАН, 408:6 (2006), 736-737.

[5] S. Bolotin, D. Treschev, "Unbounded growth of energy in nonautonomous Hamiltonian systems", Nonlinearity, 12:2 (1999), 365-388.

[6] A. Delshams, R. de la Llave, T. M. Seara, "A geometric approach to the existence of orbits with unbounded energy in generic periodic perturbations by a potential of generic geodesic flows of $\mathbb{T}^{2}$, Comm. Math. Phys., 209:2 (2000), 353-392.

[7] N. Brännström, V. Gelfreich, "Drift of slow variables in slow-fast Hamiltonian systems", Phys. D, 237:22 (2008), 2913-2921.

[8] V. Gelfreich, D. Turaev, "Unbounded energy growth in hamiltonian systems with a slowly varying parameter", Comm. Math. Phys., 283:3 (2008), 769-794.

[9] S. V. Bolotin, D. V. Treschev, "Remarks on the definition of hyperbolic tori of Hamiltonian systems", Regul. Chaotic Dyn., 5:4 (2000), 401-412.

[10] D. Treschev, O. Zubelevich, Introduction to the perturbation theory of Hamiltonian systems, Springer Monogr. Math., Springer-Verlag, Berlin, 2010.

[11] В. И. Арнольд, А.Б. Гивенталь, Симплектическая геометрия, РХД, Ижевск, 2000.

[12] Лекции по симплектической геометрии и топологии, ред. Я. Элиашберга, Л. Трейнор, МЦНМО, М., 2008; пер. с англ.: Symplectic geometry and topology. Lecture notes from the graduate summer school program (Park City, UT, USA, 1997), eds. Ya. Eliashberg, L. Traynor, Amer. Math. Soc., Providence, RI, 1999.

А. Г. Медведев (А. G. Medvedev)

Механико-математический факультет

Московского государственного университета

им. М. В. Ломоносова

E-mail: medvedev_ag@inbox.ru
Поступила в редакцию 04.03.2012 и 17.12 .2012 